\title{
QUANTITATIVE INHERITANCE OF SPIKE CHARACTERS IN CASTOR (RICINUS COMMUNIS L.)
}

\author{
Bolaji Zuluqurineen SALIHU*1 ${ }^{*}$, Isiaka Amuda YUSUF ${ }^{1}$, Benson Obukohwo APUYOR ${ }^{1}$, \\ Abolore Adijat AJADI ${ }^{2}$, Maryam Alfa KABARAINI ${ }^{1}$, Muhammad Nur ISHAQ ${ }^{1}$
}

${ }^{1}$ National Cereals Research Institute, Castor Research Programme, P.M.B. 8, Badeggi, Bida - Nigeria

${ }^{2}$ National Cereals Research Institute, Biotechnology and Central Lab., P.M.B. 8, Badeggi, Bida - Nigeria

${ }^{*}$ Corresponding author. E-mail: mobolajialabi2007@gmail.com

\begin{abstract}
Castor oil plant (Ricinus communis L.) is an important oil crop with little research attention in Nigeria. In this research, studies on quantitative inheritance of spike characters in castor, through combining ability and generation mean analyses, were carried out. This was aimed at evaluating prominent seed yield related components for selection and hybridization in castor breeding in Nigeria. The result of combining ability analysis showed significant differences for both general combining ability (GCA) and specific combining ability (SCA) for number of spikes per plant (SPP), effective spike length (ESL), capsules per spike (CPS) and 100-seeds weight. Specific combining ability was important for seed yield (SY) and seed oil content. Broad sense heritability and narrow sense heritability of $28.02 \%$ to $99.64 \%$ and $10.28 \%$ to $72.19 \%$ respectively were recorded among the traits studied. The results of scaling and joint scaling tests revealed inadequacy of simple additive-dominance model to explain the mode of gene actions for all the studied traits. The six parameters model revealed that besides the additive and dominance gene actions, epistatic interaction mechanisms have also contributed significantly to the expressions of all the characters examined. These findings suggest heterosis breeding and recurrent selections as appropriate breeding techniques for the improvement of the traits. However, where additive gene actions are to be exploited in selection program for some of the traits, the problem pose by presence of epistasis gene interactions may be overcome through delay in selection to later generation when the major operating genes will be fixable.
\end{abstract}

Keywords: castor, combining ability, generation mean, inheritance, Nigeria, quantitative, spike characters.

\section{Introduction}

Castor oil plant (Ricinus communis L.) is one of neglected African crops with high economic values [GANA \& al. 2013]. Castor oil, which is extracted from castor seed, is very critical to many industrial applications because of its ability to form many chemical derivatives [OGUNNIYI, 2006]. Castor has a wide-range of variability for qualitative and quantitative traits [WIESS, 2000]. However, the most prominent variation in castor is observed in the reproductive or spike characters such as seed size, number of capsules per spike, number of spikes, total spike length and effective spike length. These spike characters are important seed yield components in castor. RAMESH \& VENKATE (2001) reported positive relationship and direct impact for spike length, capsules per spike and 100 -seeds weight on the seed yield in castor. RAO \& al. (2006) reported that the majority of the yield parameters in castor, aside from number of seeds per capsule, are normally inherited in a quantitative manner. Several authors have reported the importance of both general combining ability (GCA) and specific combining ability (SCA) in the expression of agronomic traits in castor [TANK \& al. 2003; SOLANKI \& al. 2004; PATEL \& CHAUHAN, 2013]. RAMESH $\&$ al. (2013) reported the ratio of GCA:SCA variance in favour of non-additive gene action for all the traits evaluated in castor, except plant height to primary spike, number of nodes to 
primary spike, capsules per spike and total length of spike. However, more comprehensive genetic information for accurate decision on precise breeding methods and strategies may be obtained by combination of genetic analysis models. GRIFFING (1956a) and MATZINGER $\&$ KEMPTHORNE (1956) reported that estimates of GCA variance include, in addition to additive gene action, portions of higher order additive-type epistatic variance. Likewise, estimates of SCA variance include, in addition to dominance gene action, portions of all different types of epistatic variances. Therefore, precise decision on selection procedure may be accurately made when the decision is based on combining ability analysis in conjunction with generation mean analysis. MANIVEL (1994) reported, in a generation mean analysis experiment in castor, that simple additive - dominance model was not adequate for most of the traits in castor. MARINKOVIC \& al. (2013) recorded highly significant values of epistatic gene effects (additive $\times$ additive and dominant $\times$ dominant) for number of capsules per spike, number of seeds per plant and 100-seeds weight in two crosses of castor. SAKHARE \& al. (2017) reported duplicate type of gene action for seven out of nine characters in three crosses of castor. Among the epistatic interaction effects, both additive $\times$ dominance and additive $\times$ additive effects were reported governing number of spikes per plant and 100-seeds weight. Additive $\times$ additive epistatic interaction was predominance for effective length of spikes and additive $\times$ dominance interaction was reported for seed yield [MANIVEL, 1994].

Despite the huge economic benefits of castor, its genetic improvement in Nigeria has not been receiving much attention. In this research, studies on quantitative inheritance of spike characters through combining ability and generation mean analysis were carried out. This was aimed at evaluating prominent seed yield related components for selection and hybridization in castor breeding in Nigeria.

\section{Material and methods}

\section{Plant materials}

The plant materials used for this research were obtained from Castor Research Programme of National Cereals Research Institute (NCRI) Badeggi, Nigeria. Six (6) inbred lines developed by the Castor Research Programme of NCRI were used for the study. The six lines were developed for specific spike character such as high seed weight, high number of spikes, high number of capsules and high seed oil content. The lines include: (1) NCRICAS/Acc.005 - S5 - 2, a small seeded castor with high number of spikes per plant, (2) NCRICAS/Acc.010 - S4 - 5, a small seeded castor with high number of capsules per spike, long effective spike length and high seed oil content, (3) NCRICAS/Acc.036 - S5 - 8, a medium seeded castor with high number of spikes per plant (4) NCRICAS/Acc.001 - S6 10, a white large seeded castor, (5) NCRICAS/Acc.036M - S4 - 20, a medium seeded castor with high number of spikes per plant and high seed oil content, (6) NCRICAS/Acc.045 - S5 -4 , a black large seeded castor with high number of fruit bearing branches. 


\section{Mating design}

Partial diallel cross among the six castor parental lines were carried out. Random seed samples from the six lines were used to establish diallel crossing block between January and May 2016, using irrigation facility at the Sugarcane Research Field of NCRI Badeggi, Nigeria. Two sets of blocks were established at different planting dates (15 days interval) to synchronize the flowering periods of the parents. The selected female flowers were covered with brown envelops prior to flower opening to control the pollination. Emasculation was effected prior to anthesis by the use of forceps. The pollens were collected in the evening ( 5 - $6 \mathrm{pm}$ ) and stored in white envelope for pollination in the next day. Pollination was effected 1 to 5 days after anthesis. Partial diallel crosses were made to generate 15 hybrids. After artificial pollination, the pollination envelops were replaced and appropriate labeling were ensued. For the purpose of this research, the six parental lines were coded as P1 for NCRICAS/Acc.005-S5-2, P2 - NCRICAS/Acc.010-S4-5, P3 - NCRICAS/Acc.036-S5-8, P4 - NCRICAS/Acc.001-S6-10, P5 - NCRICAS/Acc.036M-S4-20, and P6 NCRICAS/Acc.045-S5-4.

\section{Genetic Analyses}

The six castor parental lines and their $15 \mathrm{~F} 1$ hybrids were evaluated for combining ability and heterosis between June and November, 2016. For each set of the crosses, 45 plant samples of F1 and parents were evaluated. The entries were laid out on Randomized Complete Block Design with three replications. The plot size was $3 \mathrm{~m}$ by $1.5 \mathrm{~m}$ with Interrow and intra-row spaces of $75 \mathrm{~cm}$. Two seeds per hole were planted and later thinned to one seedling per hole at four weeks after planting. Morphological data were taken according to Indian castor descriptor (INDIA, 2004). The parameters observed were: spike per plant, effective length of spike, number of capsules per spike, 100-seeds weight, seed yield and seed-oil content.

Three crosses $(\mathrm{P} 1 \times \mathrm{P} 4, \mathrm{P} 2 \times \mathrm{P} 6$ and $\mathrm{P} 5 \times \mathrm{P} 6)$ with high overall specific combining ability and high heterosis for seed yield over the better parents were advanced to $\mathrm{F}_{2}$ and backcrosses $(\mathrm{BC})$ with the parents were made to generate $\mathrm{F}_{1} \mathrm{BC}_{1}$ and $\mathrm{F}_{1} \mathrm{BC}_{2}$ from parent 1 and parent 2 respectively. Seeds of successful crosses were harvested, processed and 20 individuals were self-pollinated to generate $F_{2}$ seeds. For the backcrosses $(B C), F_{1}$ plants were crossed with the two parents using the same procedure described above. The development of filial generation was done between June and November, 2016.

In 2017 growing season, the three crosses and their filial generations were evaluated for generation mean analysis on Compact Family Block Design with three replicated plots at NCRI Badeggi-Nigeria. Plant spacing, cultural practices and data taken described earlier were adopted.

\section{Data analysis}

General analysis of variance was carried out to test for significant of variability among the six parents and their hybrids. Combining ability analysis and variance component estimates were computed following the procedure of Plant Breeding Tools (PBTools, 1.3) for Griffing Diallel Method II. Ranking for overall general and overall specific combining ability was carried out according to SHIVANNA (2008).

Heterosis over mid parent (MP) and better parent (BP) were computed from mean of the treatments according to SHIVANNA (2008). 
The standard error (SE) for the estimated heterosis was determined using mean squares due to error (MSe) as described by SHIVANNA (2008). The significance of the heterosis was determined using T-test.

$\mathrm{SE}(\mathrm{MP})=\sqrt{ }(2 \times \mathrm{MSe} / 3 \mathrm{r})$ for testing heterosis over MP

$\mathrm{SE}(\mathrm{BP})=\sqrt{ }(2 \times \mathrm{MSe} / \mathrm{r})$ for testing heterosis over BP

Data from the two parents, $F_{1}, F_{2}, F_{1} B_{1}$ and $F_{1} B_{2}$ of all crosses was subjected to analysis of variance following the procedure of Statistical Tools for Agricultural Research (STAR 2.0.1, 2014) and the parameters that showed significant difference were used for scaling and joint scaling tests as described by DAVID (2006). Six parameters model of generation mean analysis was done according to TNAUSTAT General Breeding Procedure and DOSBox 0.74. Twelve (12) plant samples each of the two parents and $\mathrm{F}_{1}$ populations; 120 individual plants of $F_{2}$ populations; 21 plant samples each of $F_{1} B_{1}$ and $F_{1} B_{2}$ populations were used for the analysis.

\section{Results}

The mean square values from analysis of variance (ANOVA) for the spike characters of the parents and their hybrids are shown in Table 1. The analysis of variance showed significant differences for all the studied traits among the entries. Analysis of variance for combining ability showed significant differences for both general combining ability (GCA) and specific combining ability (SCA) for number of spikes per plant (SPP), effective spike length (ESL), capsules per spike (CPS) and 100-seeds weight. Only specific combining ability was significant for seed yield (SY) and seed oil content (Table 2). Genetic variances due to general combining ability (GCA) and specific combining ability (SCA) ability are also presented in Table 2. Variance due to general combining ability was found to be larger than variance due to specific combining ability for spike per plant and 100 seeds weight. Higher SCA variances were recorded for effective spike length, capsules per spike, seed yield and seed oil content. Broad sense heritability and narrow sense heritability ranged from $28.02 \%$ to $99.64 \%$ and $10.28 \%$ to $72.19 \%$ respectively were recorded among the traits studied (Table 2). The highest broad sense heritability (99.64\%) and highest narrow sense heritability $(72.19 \%)$ were observed in 100 -seeds weight. Broad sense heritability of $53.15 \%$ and narrow sense heritability of $10.28 \%$ were recorded for seed yield per hectare (Table 2).

Table 1. Mean squares from analysis of variance of six castor parents and their hybrids for seed yield and spike characters

\begin{tabular}{|c|c|c|c|c|c|c|c|}
\hline $\begin{array}{c}\text { Sources } \\
\text { of } \\
\text { Variation }\end{array}$ & DF & $\begin{array}{l}\text { Spike } \\
\text { per } \\
\text { plant } \\
\text { (SPP) }\end{array}$ & $\begin{array}{l}\text { Effective } \\
\text { Spike } \\
\text { Length } \\
\text { (ESL) }\end{array}$ & $\begin{array}{c}\text { Capsules } \\
\text { per Spike } \\
\text { (CPS) }\end{array}$ & $\begin{array}{c}\text { Seed } \\
\text { Weight } \\
(\mathrm{SW})(\mathrm{g})\end{array}$ & $\begin{array}{l}\text { Seed Yield } \\
\text { (SY) (kg/ha) }\end{array}$ & $\begin{array}{r}\text { Seed Oil } \\
\text { Content } \\
(\text { SOC })(\%)\end{array}$ \\
\hline $\begin{array}{c}\text { All } \\
\text { Genotypes }\end{array}$ & 20 & $22.28 * *$ & $135.95 * *$ & $1366.94 * *$ & $462.58 * *$ & $647322.58 * *$ & $247.98 * *$ \\
\hline Parents & 5 & $16.26 \mathrm{~ns}$ & $131.41 * *$ & $2022.69 * *$ & $996.96 * *$ & $392915.21 * *$ & $313.29 * *$ \\
\hline Crosses & 14 & $24.65 * *$ & $142.34 * *$ & $1224.12 * *$ & $300.61 * *$ & $674379.92 * *$ & $106.27 * *$ \\
\hline $\begin{array}{l}\text { Parents vs } \\
\text { Crosses }\end{array}$ & 1 & $7.95 \mathrm{~ns}$ & $36.06^{*}$ & $114.72 *$ & $66.91 \mathrm{~ns}$ & $1540557.21 * *$ & $1929.33 * *$ \\
\hline
\end{tabular}


BOLAJI ZULUQURINEEN SALIHU \& al.

Table 2. Mean square and variances for general and specific combining ability for seed yield and spike characters in castor

\begin{tabular}{ccccccc}
\hline Characters & GCA & SCA & $\mathbf{V g}$ & $\mathbf{V} \boldsymbol{\boldsymbol { h } ^ { 2 }}$ & $\boldsymbol{H}^{\mathbf{2}}$ \\
\hline SPP & $19.93^{* *}$ & $3.65^{*}$ & 2.32 & 1.62 & 17.20 & 31.70 \\
ESL & $89.61^{*}$ & $29.45^{* *}$ & 7.45 & 26.42 & 21.11 & 28.02 \\
CPS & $1041.20^{*}$ & $259.57^{* *}$ & 97.71 & 176.15 & 27.23 & 86.22 \\
$100 \mathrm{SW}$ & $540.05^{* *}$ & $25.57^{* *}$ & 64.73 & 25.38 & 72.19 & 99.64 \\
SY & $284785.50 \mathrm{~ns}$ & $181456.80^{* *}$ & 12916.21 & 181380.48 & 10.28 & 53.15 \\
SOC & $75.28 \mathrm{~ns}$ & $84.67^{* *}$ & 0.02 & 81.21 & 56.82 & 96.91 \\
\hline
\end{tabular}

General combining ability (GCA) effects of the parents on all the studied characters are presented in Table 3. All parents showed significant GCA effects for capsules per spike, 100-seeds weight, seed yield and seed oil content. All the parents, except P4, showed significant GCA effect for spikes per plant. The highest positive GCA effects for spikes per plant, effective spike length, 100 seed weight, and seed oil content were recorded in P1, P2, P6, and P5 respectively. Specific combining ability (SCA) effects for effective spike length was significant in eight out of 15 hybrids evaluated (Table 4). Six hybrids showed significant SCA effects for capsules per spike (Table 4). All hybrids, except P2xP3, showed significant effects for 100-seeds weight, however the maximum positive effect was observed in $\mathrm{P} 4 \mathrm{xP} 6$ (Table 4). The highest positive SCA effects for seed yield and seed oil content was observed in hybrid P2xP6 and P1xP5 respectively (Table 4). Ranking status of each of the parents and hybrids, to determine good general and specific combiners across the studied character, is presented in Table 5. The result showed high overall general combining ability ( $>$ Norm) only in three parents, namely P1 (Score 24), P2 (Score 24) and P5 (Score 27). The best overall general combiner was parent $\mathrm{P} 5$ which has the highest total score. Other three parents $(\mathrm{P} 3$, $\mathrm{P} 4$, and P6) were found to be low (L) overall general combiners for the characters evaluated. Eight hybrids namely; P1XP2 (Score 54), P1XP3 (Score 64), P1XP4 (Score 57), P1XP5 (Score 67), P2XP6 (Score 64), P3XP5 (Score 55), P4XP5 (Score 55) and P5XP6 (Score 62) were found to be good specific combiners for the traits studied. The highest overall specific combiner was hybrid P1XP3 with the highest total score. The least average specific combiner (Score 26) among the hybrid was P1XP6.

Table 3. Estimates of general combining ability effects $(g i)$ of each parent for seed yield and spike

\begin{tabular}{ccccccc}
\hline Parents & $\begin{array}{c}\text { Spike per } \\
\text { plant }\end{array}$ & $\begin{array}{c}\text { Effective } \\
\text { Spike Length }\end{array}$ & $\begin{array}{c}\text { Capsules } \\
\text { per Spike }\end{array}$ & $\begin{array}{c}\text { Seed } \\
\text { Weight (g) }\end{array}$ & $\begin{array}{c}\text { Seed Yield } \\
\text { (kg/ha) }\end{array}$ & $\begin{array}{c}\text { Seed Oil } \\
\text { Content (\%) }\end{array}$ \\
\hline P1 & $2.29^{* *}$ & $-1.18^{* *}$ & $10.21^{* *}$ & $-7.52^{* *}$ & $251.36^{* *}$ & $-4.43^{* *}$ \\
P2 & $-2.03^{* *}$ & $5.75^{* *}$ & $15.46^{* *}$ & $-6.79^{* *}$ & $-86.14^{*}$ & $3.21^{* *}$ \\
P3 & $0.38^{* *}$ & $-1.30^{* *}$ & $-5.65 \mathrm{~ns}$ & $-4.17^{* *}$ & $-147.63^{* *}$ & $-1.37^{* *}$ \\
P4 & $-0.03 \mathrm{~ns}$ & $-1.16^{* *}$ & $-5.47 \mathrm{~ns}$ & $8.32^{* *}$ & $128.34^{* *}$ & $1.14^{* *}$ \\
P5 & $0.83^{* *}$ & $1.36^{* *}$ & $1.45 \mathrm{~ns}$ & $-2.25^{* *}$ & $97.69^{* *}$ & $3.55^{* *}$ \\
P6 & $-1.56^{* *}$ & $-4.18^{* *}$ & $-15.76^{* *}$ & $12.09^{* *}$ & $-244.35^{* *}$ & $-2.52^{* *}$ \\
\hline SE $(g i)$ & 0.39 & 0.55 & 2.85 & 0.13 & 22.81 & 0.62 \\
\hline
\end{tabular}


QUANTITATIVE INHERITANCE OF SPIKE CHARACTERS IN CASTOR...

Table 4. Estimates of specific combining ability effects $(\mathrm{gi})$ of 15 castor hybrids for seed yield and spike characters in castor

\begin{tabular}{lcccccc}
\hline Hybrids & $\begin{array}{c}\text { Spike per } \\
\text { plant }\end{array}$ & $\begin{array}{c}\text { Effective } \\
\text { Spike Length }\end{array}$ & $\begin{array}{c}\text { Capsules } \\
\text { per Spike }\end{array}$ & $\begin{array}{c}\text { Seed } \\
\text { Weight }(\mathrm{g})\end{array}$ & $\begin{array}{c}\text { Seed Yield } \\
(\mathrm{kg} / \mathrm{ha})\end{array}$ & $\begin{array}{c}\text { Seed Oil } \\
\text { Content }(\%)\end{array}$ \\
\hline P1XP2 & $0.24 \mathrm{~ns}$ & $-2.33 \mathrm{~ns}$ & $-3.71 \mathrm{~ns}$ & $2.22^{* *}$ & $107.80^{* *}$ & $5.34^{* *}$ \\
P1XP3 & $-0.25 \mathrm{~ns}$ & $-1.26 \mathrm{~ns}$ & $12.97 \mathrm{~ns}$ & $2.28^{* *}$ & $231.09^{* *}$ & $13.47^{* *}$ \\
P1XP4 & $2.89^{* *}$ & $0.65 \mathrm{~ns}$ & $23.46^{* *}$ & $-3.45^{* *}$ & $439.25^{* *}$ & $5.17 * *$ \\
P1XP5 & $2.16^{*}$ & $6.63^{* *}$ & $-7.23 \mathrm{~ns}$ & $0.81^{*}$ & $175.20^{* *}$ & $13.92^{* *}$ \\
P1XP6 & $-0.33 \mathrm{~ns}$ & $-4.99^{* *}$ & $-16.96^{*}$ & $-7.96^{* *}$ & $-346.27 * *$ & $-4.34 * *$ \\
P2XP3 & $-0.92 \mathrm{~ns}$ & $-1.45 \mathrm{~ns}$ & $-19.75^{*}$ & $0.24 \mathrm{~ns}$ & $-74.97 * *$ & $-2.14 \mathrm{~ns}$ \\
P2XP4 & $-1.29 \mathrm{~ns}$ & $5.89^{* *}$ & $4.21 \mathrm{~ns}$ & $-2.48^{* *}$ & $94.97 * *$ & $2.46 \mathrm{~ns}$ \\
P2XP5 & $-2.42^{*}$ & $-7.60^{* *}$ & $-15.96^{*}$ & $1.52^{* *}$ & $-287.79^{* *}$ & $4.47^{* *}$ \\
P2XP6 & $1.67 \mathrm{~ns}$ & $12.76^{* *}$ & $38.51^{* *}$ & $-6.79^{* *}$ & $891.17^{* *}$ & $-0.63 \mathrm{~ns}$ \\
P3XP4 & $1.11 \mathrm{~ns}$ & $1.17 \mathrm{~ns}$ & $1.52 \mathrm{~ns}$ & $-6.99^{* *}$ & $-153.91^{* *}$ & $6.33^{* *}$ \\
P3XP5 & $-1.27 \mathrm{~ns}$ & $-0.71 \mathrm{~ns}$ & $-5.13 \mathrm{~ns}$ & $6.56^{* *}$ & $-133.78^{* *}$ & $7.25^{* *}$ \\
P3XP6 & $-0.43 \mathrm{~ns}$ & $1.29 \mathrm{~ns}$ & $7.86 \mathrm{~ns}$ & $-2.28^{* *}$ & $13.50 \mathrm{~ns}$ & $3.99^{*}$ \\
P4XP5 & $0.67 \mathrm{~ns}$ & $-2.74^{*}$ & $2.61 \mathrm{~ns}$ & $3.67 * *$ & $564.54 * *$ & $-4.27 * *$ \\
P4XP6 & $-1.72 \mathrm{~ns}$ & $-6.19^{* *}$ & $-16.76^{*}$ & $6.62^{* *}$ & $-519.95^{* *}$ & $-0.76 \mathrm{~ns}$ \\
P5XP6 & $2.78^{* *}$ & $5.84^{* *}$ & 6.66 & $-3.67 * *$ & $524.27 * *$ & $2.52 \mathrm{~ns}$ \\
\hline SE $\left(\mathrm{S}_{i i}\right)$ & 0.87 & 1.28 & 6.79 & 0.31 & 16.45 & 1.35 \\
\hline
\end{tabular}

Table 5. Overall general combining and specific combining ability status of six parents and their hybrids for seed yield and spike characters in castor

\begin{tabular}{ccccccccc}
\hline Entries & SPP & SL & CPS & SW & SY & SOC & Total & Rank \\
\hline P1 & 6 & 5 & 5 & 1 & 6 & 1 & 24 & $\mathrm{H}$ \\
P2 & 2 & 6 & 6 & 2 & 3 & 5 & 24 & $\mathrm{H}$ \\
P3 & 4 & 3 & 3 & 3 & 2 & 3 & 18 & $\mathrm{~L}$ \\
P4 & 3 & 2 & 2 & 5 & 5 & 4 & 21 & $\mathrm{~L}$ \\
P5 & 5 & 4 & 4 & 4 & 4 & 6 & 27 & $\mathrm{H}$ \\
P6 & 1 & 1 & 1 & 6 & 1 & 2 & 12 & $\mathrm{~L}$ \\
\hline Mean (Norm) & & & & & & & 21 & \\
\hline P1XP2 & 9 & 10 & 4 & 12 & 8 & 11 & 54 & $\mathrm{H}$ \\
P1XP3 & 8 & 6 & 13 & 11 & 12 & 14 & 64 & $\mathrm{H}$ \\
P1XP4 & 15 & 5 & 14 & 5 & 11 & 7 & 57 & $\mathrm{H}$ \\
P1XP5 & 13 & 13 & 8 & 9 & 9 & 15 & 67 & $\mathrm{H}$ \\
P1XP6 & 7 & 3 & 2 & 1 & 7 & 2 & 22 & $\mathrm{~L}$ \\
P2XP3 & 5 & 4 & 5 & 8 & 4 & 3 & 29 & $\mathrm{~L}$ \\
P2XP4 & 3 & 8 & 12 & 6 & 10 & 10 & 49 & $\mathrm{~L}$ \\
P2XP5 & 1 & 1 & 1 & 10 & 2 & 8 & 23 & $\mathrm{~L}$ \\
P2XP6 & 12 & 15 & 15 & 3 & 15 & 4 & 64 & $\mathrm{H}$ \\
P3XP4 & 11 & 11 & 7 & 2 & 3 & 12 & 46 & $\mathrm{~L}$ \\
P3XP5 & 4 & 12 & 6 & 14 & 6 & 13 & 55 & $\mathrm{H}$ \\
P3XP6 & 6 & 9 & 9 & 7 & 5 & 9 & 45 & $\mathrm{~L}$ \\
P4XP5 & 10 & 7 & 11 & 13 & 13 & 1 & 55 & $\mathrm{H}$ \\
P4XP6 & 2 & 2 & 3 & 15 & 1 & 5 & 28 & $\mathrm{~L}$ \\
P5XP6 & 14 & 14 & 10 & 4 & 14 & 6 & 62 & $\mathrm{H}$ \\
\hline Mean (Norm) & & & & & & 49 & \\
\hline
\end{tabular}


The heterosis exhibited by the 15 hybrids over the mid parent (MP) and better parent (BP) values for the traits evaluated are shown in Table 6. None of the crosses, except P2XP4, recorded negative heterosis over mid-parents for number of spikes per plant (Table 6). Heterosis over better parent ranged between $-48.62 \%$ in P3XP6 and $34.58 \%$ in P4XP5. Four crosses (P1XP4, P1XP5, P4XP5 and P5XP6) showed significant positive heterosis over better parent for number of spikes per plant. All the crosses recorded significant heterosis over both mid parents for effective spike length, however, only crosses P1XP5, P3XP5, P4XP5 and P5XP6 showed significant positive heterosis over better parent. Positive significant heterosis over the mid parent was found for seed weight in all the crosses except P1XP2 and P2XP3. Negative heterosis over better parent were observed for seed weight in all the crosses, except P2XP5 and P3XP5. All the crosses recorded positive mid parent heterotic values for seed yield, except P4XP6. Eight hybrids (P1XP3, P1XP4, P1XP5, P2XP4, P2XP6, P3XP5, P4XP5 and P5XP6) were found to have positive and significant heterosis over the better parent seed yield. Positive and significant heterosis over mid parents for seed oil content was recorded in all the crosses, except cross P4XP5 and P4XP6. Out of the fifteen hybrids, only three crosses (P1XP6, P2XP3 and P2XP6) recorded negative heterosis over better parent for seed oil content. The highest desirable heterosis (73.32 \%) over better parent was observed in P1XP3.

The data collected from the generations of three crosses were subjected to individual analysis of variance. Significant differences were observed for all the traits studied in all the three crosses evaluated (Table 7). The mean performances of the generations from the three crosses are presented in Table 8. Average number of spikes per plant for $F_{1}$ ranged from 3.6 to $12 \cdot 1$ among the crosses. The parental mean for number of spikes per plant was from $3 \cdot 3$ to 15 . In all the crosses, $\mathrm{F}_{2}$ values for spikes per plant was only less than $\mathrm{F}_{1}$ mean in Cross II. In $\mathrm{BC}_{1}$, only Cross III recorded greater spikes per plant than its recurrent parent. In $\mathrm{BC}_{2}$, Lesser mean to recurrent parents were observed in Cross I and Cross II while in Cross III greater mean than recurrent parent was recorded. For seed yield, the parental mean ranged from $125 \cdot 26 \mathrm{~g}$ to 379.98 g per plant (Table 8 ). The $F_{1}$ recorded seed yield range between $62.60 \mathrm{~g}$ (Cross III) and 153.74 $\mathrm{g}$ (Cross II). The $\mathrm{F}_{2}$ yield varied from $24.43 \mathrm{~g}$ (Cross III) to $84 \cdot 89 \mathrm{~g}$ (Cross I). The $\mathrm{BC}_{1}$ and $\mathrm{BC}_{2}$ recorded yield ranging from $77 \cdot 84 \mathrm{~g}$ to $189 \cdot 57 \mathrm{~g}$ and $48.86 \mathrm{~g}$ to $140 \cdot 13 \mathrm{~g}$ respectively. Among all the three crosses, none of the $\mathrm{F}_{1}$ recorded seed yield greater than their better parents. The oil content of the parent ranged from $24 \%$ to $50 \cdot 33 \%$ (Table 8 ). For $F_{1}$, the seed oil content ranged from $41.67 \%$ to $54.00 \%$. In the $\mathrm{F}_{2}$, the oil content ranged from $32.67 \%$ to $43.33 \%$ among the three crosses. Out of the three crosses, only $\mathrm{F}_{1}$ of Cross III recorded oil content greater than its better parent. The $\mathrm{F}_{2}$ oil content was lesser than $\mathrm{F}_{1}$ mean in all the three crosses. The $\mathrm{BC}_{1}$ mean was greater than recurrent parent values only in Cross I. The $\mathrm{BC}_{2}$ recorded oil content greater than that of recurrent parent only in Cross III. 
QUANTITATIVE INHERITANCE OF SPIKE CHARACTERS IN CASTOR...

Table 6. Heterosis over Mid-Parents (MP) and Better Parents (BP) for Seed Yield and Spike Characters in Castor

\begin{tabular}{|c|c|c|c|c|c|c|c|c|c|c|c|c|}
\hline \multirow[b]{2}{*}{ Hybrids } & \multicolumn{2}{|c|}{$\begin{array}{c}\text { Spikes per Plant } \\
\text { (RPP) }\end{array}$} & \multicolumn{2}{|c|}{$\begin{array}{c}\text { Effective Spike } \\
\text { Length (RL) }\end{array}$} & \multicolumn{2}{|c|}{$\begin{array}{c}\text { Capsules per Spike } \\
\text { (CPR) }\end{array}$} & \multicolumn{2}{|c|}{$\begin{array}{c}\text { 100-Seeds Weight } \\
\text { (SW) }\end{array}$} & \multicolumn{2}{|c|}{ Seed Yield (SY) } & \multicolumn{2}{|c|}{$\begin{array}{l}\text { Seed Oil Content } \\
\text { (SOC) }\end{array}$} \\
\hline & MP & $\mathrm{BP}$ & $\mathrm{MP}$ & $\overline{\mathrm{BP}}$ & $\mathrm{MP}$ & $\mathrm{BP}$ & $\mathrm{MP}$ & $\mathrm{BP}$ & $\mathrm{MP}$ & $\overline{\mathrm{BP}}$ & MP & $\mathrm{BP}$ \\
\hline P1XP2 & $100.35 * *$ & $-5.50 * *$ & $16.53 * *$ & $-4.22 * *$ & $3.00 \mathrm{~ns}$ & $-11.82 * *$ & $-0.08 n s$ & $-9.52 * *$ & $101.37 * *$ & $1.38 \mathrm{~ns}$ & $94.36 * *$ & $5.77 \mathrm{~ns}$ \\
\hline P1XP3 & $0.00 \mathrm{~ns}$ & $-2.76 * *$ & $4.33 * *$ & $-2.96 * *$ & $70.15^{* *}$ & $-1.17 n s$ & $19.87 * *$ & $-8.94 * *$ & $95.22 * *$ & $16.96 *$ & $103.34 * *$ & $73.32 * *$ \\
\hline P1XP4 & $104.86 * *$ & $19.50 * *$ & $5.38 * *$ & $-10.95 * *$ & $138.34 * *$ & $12.14 * *$ & $77.80 * *$ & $-48.85 * *$ & $73.60 * *$ & $28.39 *$ & $78.30 * *$ & $6.16 \mathrm{~ns}$ \\
\hline P1XP5 & $58.80 * *$ & $27.83 * *$ & $17.12 * *$ & $7.72 * *$ & $-1.39 \mathrm{~ns}$ & $-14.29 * *$ & $24.37 * *$ & $-4.36 * *$ & $109.75 * *$ & $12.46 *$ & $125.91 * *$ & $35.96 * *$ \\
\hline P1XP6 & $209.84 * *$ & $-5.50 * *$ & $44.63 * *$ & $-23.53 * *$ & $154.05 * *$ & $-61.70 * *$ & $72.45 * *$ & $-62.27 * *$ & $92.47 * *$ & $-16.64 *$ & $39.06 * *$ & $-14.04 *$ \\
\hline P2XP3 & $17.67 * *$ & $-46.03 * *$ & $14.26 * *$ & $-12.66^{* *}$ & $50.73 * *$ & $-25.05 * *$ & $-0.38 *$ & $-16.42 * *$ & $15.17 *$ & $-3.22 \mathrm{~ns}$ & $51.61 * *$ & $-3.21 n s$ \\
\hline P2XP4 & $-11.66 * *$ & $-28.57 * *$ & $26.50 * *$ & $-12.15 * *$ & $125.15^{* *}$ & $-9.31 * *$ & $74.53 * *$ & $-44.55 * *$ & $84.72 * *$ & $25.75 *$ & $24.26 * *$ & $13.57 *$ \\
\hline P2XP5 & $23.67 * *$ & $-27.54 * *$ & $9.48 * *$ & $-17.24 * *$ & $-19.37 * *$ & $-40.00 * *$ & $22.52 * *$ & $4.04 * *$ & $8.62 \mathrm{~ns}$ & $1.99 \mathrm{~ns}$ & $28.78 * *$ & $16.45^{*}$ \\
\hline P2XP6 & $54.64 * *$ & $0.00 \mathrm{~ns}$ & $121.50 * *$ & $-3.74 * *$ & $855.89 * *$ & $23.37 * *$ & $69.16 * *$ & $-59.13 * *$ & $166.70 * *$ & $129.45 * *$ & $10.84 *$ & $-2.42 \mathrm{~ns}$ \\
\hline P3XP4 & $62.00 * *$ & $-8.10 * *$ & $9.99 * *$ & $-0.07 \mathrm{~ns}$ & $17.77 * *$ & $-4.60 *$ & $33.67 * *$ & $-49.38 * *$ & $2.17 \mathrm{~ns}$ & $-17.24 *$ & $69.62 * *$ & $18.49 *$ \\
\hline P3XP5 & $10.35 * *$ & $-13.61 * *$ & $11.16^{* *}$ & $9.93 * *$ & $6.57 *$ & $31.56 * *$ & $52.57 * *$ & $50.73 * *$ & $31.00 * *$ & $17.24 *$ & $80.61 * *$ & $27.53^{*}$ \\
\hline P3XP6 & $73.22 * *$ & $-48.62 * *$ & $56.55^{* *}$ & $-11.00 * *$ & $233.30 * *$ & $-13.50 * *$ & $87.19 * *$ & $-46.09 * *$ & $9.22 *$ & $-21.04 *$ & $54.70 * *$ & $12.19 *$ \\
\hline P4XP6 & $63.93 * *$ & $-14.29 * *$ & $25.41 * *$ & $-21.52 * *$ & $33.30 * *$ & $-57.29 * *$ & $22.02 * *$ & $-7.21 * *$ & $-24.07 * *$ & $-55.53 * *$ & $7.20 \mathrm{~ns}$ & $3.25 \mathrm{~ns}$ \\
\hline P4XP5 & $85.71 * *$ & $34.58 * *$ & $9.74 * *$ & $0.82 *$ & $62.56 * *$ & $-12.00 * *$ & $116.82 * *$ & $-18.88 * *$ & $131.07 * *$ & $27.53 * *$ & $2.70 \mathrm{~ns}$ & $1.60 \mathrm{~ns}$ \\
\hline P5XP6 & $182.51 * *$ & $7.04 * *$ & $79.28 * *$ & $3.07 * *$ & $303.57 * *$ & $-30.00 * *$ & $94.80 * *$ & $-44.58 * *$ & $99.73 * *$ & $61.34 * *$ & $18.81 * *$ & $15.68 *$ \\
\hline SE & 0.28 & 0.16 & 0.40 & 0.23 & 2.17 & 1.26 & 0.11 & 0.06 & 3.01 & 4.99 & 2.88 & 4.61 \\
\hline
\end{tabular}

* - Significant at $\mathrm{p}<0.05, * *$ - Significant at $\mathrm{p}<0.01, \mathrm{~ns}-$ not significant, $\mathrm{t}$-value $(0.05, \mathrm{df}=15)=2.145, \mathrm{t}-\mathrm{value}(0.01, \mathrm{df}=14)=2.977$ 
BOLAJI ZULUQURINEEN SALIHU \& al.

Table 7. Mean Square Values from Individual Analysis of Variance for Seed Yield and Spike Characters of three Crosses of Castor

\begin{tabular}{lccc}
\hline Parameters & P1×P4 & P2×P6 & P5×P6 \\
\hline Number of Spike Per Plant & $8.719^{*}$ & $20.50^{* *}$ & $44.30^{* *}$ \\
Effective Length of Spike (cm) & $311.713^{* *}$ & $994.135^{* *}$ & $1080.63^{* *}$ \\
Number of Capsules Per Spike & $8892.39^{*}$ & $16769.34 * *$ & $10127.56^{* *}$ \\
100 Seed Weight (g) & $3759.71^{* *}$ & $8597.24 * *$ & $4464.19^{* *}$ \\
Seed Yield Per Plant (g) & $187356.94 *$ & $119682.72^{* *}$ & $94502.23^{* *}$ \\
Seed Oil Content $(\%)$ & $222.09^{* *}$ & $71.12^{* *}$ & $41.66^{* *}$ \\
\hline
\end{tabular}

Table 8. Mean Performance and Variance for Seed Yield and Spike Characters among Generations of three Crosses of Castor

\begin{tabular}{|c|c|c|c|c|c|c|c|}
\hline & \multirow[b]{2}{*}{ Generations } & \multicolumn{2}{|c|}{$\mathrm{P} 1 \times \mathrm{P} 4$} & \multicolumn{2}{|c|}{$\mathrm{P} 2 \times \mathrm{P} 6$} & \multicolumn{2}{|c|}{$\mathrm{P} 5 \times \mathrm{P} 6$} \\
\hline & & Mean \pm S.E & Variance & Mean \pm S.E & $\overline{\text { Variance }}$ & Mean \pm S.E & Variance \\
\hline \multirow{8}{*}{$\begin{array}{l}\text { Number } \\
\text { of Spike } \\
\text { per Plant }\end{array}$} & $\mathrm{P}_{1}$ & $15.00 \pm 0.26^{\mathrm{ab}}$ & 0.77 & $12.30 \pm 0.21^{\mathrm{a}}$ & 0.46 & $9.70 \pm 0.33 \mathrm{a}$ & 0.11 \\
\hline & $\mathrm{P}_{2}$ & $9.60 \pm 0.16^{\mathrm{b}}$ & 0.33 & $6.40 \pm 0.16^{b}$ & 0.27 & $3.30 \pm 0.30^{\mathrm{b}}$ & 0.09 \\
\hline & F1 & $12.10 \pm 0.90^{\mathrm{a}}$ & 0.81 & $6.30 \pm 0.15^{\mathrm{bc}}$ & 0.23 & $3.60 \pm 0.16^{\mathrm{b}}$ & 0.03 \\
\hline & $\mathrm{F}_{2}$ & $12.64 \pm 0.17^{\mathrm{ab}}$ & 5.03 & $3.48 \pm 0.07^{\mathrm{d}}$ & 0.76 & $6.00 \pm 0.11^{\mathrm{b}}$ & 0.32 \\
\hline & $\mathrm{BC}_{1}$ & $12.35 \pm 0.24^{\mathrm{b}}$ & 3.06 & $6.85 \pm 0.23^{b}$ & 1.08 & $12.50 \pm 0.41^{\mathrm{a}}$ & 0.17 \\
\hline & $\mathrm{BC}_{2}$ & $9.55 \pm 0.42^{\mathrm{ab}}$ & 2.76 & $3.75 \pm 0.17^{\mathrm{cd}}$ & 0.62 & $12.40 \pm 0.45^{\mathrm{a}}$ & 0.20 \\
\hline & Mean & 2.83 & & 1.88 & & 2.52 & \\
\hline & SD & 1.89 & & 1.10 & & 1.71 & \\
\hline \multirow{8}{*}{$\begin{array}{l}\text { Effective } \\
\text { Spike } \\
\text { Length } \\
(\mathrm{cm})\end{array}$} & $\mathrm{P}_{1}$ & $20.49 \pm 1.19^{\mathrm{a}}$ & 1.44 & $26.49 \pm 0.96^{\mathrm{b}}$ & 3.31 & $29.05 \pm 0.87^{\mathrm{a}}$ & 0.76 \\
\hline & $\mathrm{P}_{2}$ & $10.11 \pm 0.31^{\mathrm{bc}}$ & 0.09 & $11.18 \pm 0.55^{\mathrm{d}}$ & 3.09 & $13.18 \pm 0.55^{\mathrm{d}}$ & 0.31 \\
\hline & $\mathrm{F} 1$ & $13.14 \pm 0.84^{\mathrm{b}}$ & 0.01 & $38.401 .99^{\mathrm{a}}$ & 3.82 & $25.00 \pm 1.92^{\mathrm{b}}$ & 0.71 \\
\hline & $\mathrm{F}_{2}$ & $8.78 \pm 0.39^{c}$ & 0.16 & $18.44 \pm 0.67^{\mathrm{c}}$ & 47.13 & $11.43 \pm 0.34^{\mathrm{d}}$ & 6.92 \\
\hline & $\mathrm{BC}_{1}$ & $10.11 \pm 0.57^{\mathrm{bc}}$ & 0.33 & $20.64 \pm 0.60^{\mathrm{c}}$ & 37.29 & $18.74 \pm 1.73^{\mathrm{c}}$ & 3.02 \\
\hline & $\mathrm{BC}_{2}$ & $12.96 \pm 0.78^{\mathrm{b}}$ & 0.61 & $17.88 \pm 1.78^{\mathrm{c}}$ & 43.91 & $19.93 \pm 1.88^{c}$ & 3.56 \\
\hline & Mean & 10.21 & & 19.68 & & 14.66 & \\
\hline & SD & 4.90 & & 8.52 & & 7.21 & \\
\hline \multirow{2}{*}{$\begin{array}{c}\text { Number } \\
\text { of }\end{array}$} & $\mathrm{P}_{1}$ & $77.00 \pm 1.14^{\mathrm{a}}$ & 12.89 & $49.30 \pm 3.91^{\mathrm{bc}}$ & 5.29 & $91.00 \pm 2.40^{\mathrm{a}}$ & 5.78 \\
\hline & $\mathrm{P}_{2}$ & $20.00 \pm 0.36^{\mathrm{d}}$ & 1.33 & $19.60 \pm 3.08^{\mathrm{d}}$ & 9.49 & $21.60 \pm 3.08^{\mathrm{d}}$ & 9.49 \\
\hline \multirow{6}{*}{$\begin{array}{c}\text { Capsules } \\
\text { per } \\
\text { Spike }\end{array}$} & $\mathrm{F} 1$ & $35.90 \pm 1.91^{\mathrm{c}}$ & 16.32 & $121.60 \pm 8.58^{\mathrm{a}}$ & 3.58 & $52.90 \pm 4.33^{\mathrm{b}}$ & 8.81 \\
\hline & $\mathrm{F}_{2}$ & $25.90 \pm 1.30^{\mathrm{d}}$ & 220.53 & $35.11 \pm 1.73^{\mathrm{c}}$ & 93.00 & $24.04 \pm 0.75^{\mathrm{d}}$ & 50.57 \\
\hline & $\mathrm{BC}_{1}$ & $55.40 \pm 4.04^{\mathrm{b}}$ & 126.36 & $62.85 \pm 3.83^{\mathrm{b}}$ & 14.66 & $38.65 \pm 2.61^{\mathrm{c}}$ & 26.84 \\
\hline & $\mathrm{BC}_{2}$ & $21.90 \pm 2.28^{c}$ & 104.51 & $39.35 \pm 4.58^{c}$ & 21.03 & $40.00 \pm 3.51^{\mathrm{c}}$ & 22.32 \\
\hline & Mean & 29.71 & & 42.57 & & 31.77 & \\
\hline & SD & 20.24 & & 28.06 & & 18.84 & \\
\hline $100-$ & $P_{1}$ & $16.44 \pm 0.93^{\mathrm{b}}$ & 8.79 & $11.59 \pm 0.27^{\mathrm{d}}$ & 0.72 & $13.59 \pm 0.28^{\mathrm{f}}$ & 2.08 \\
\hline Seeds & $\mathrm{P}_{2}$ & $58.28 \pm 0.72^{\mathrm{a}}$ & 5.26 & $51.24 \pm 0.65^{\mathrm{a}}$ & 4.29 & $53.34 \pm 0.65^{\mathrm{a}}$ & 1.43 \\
\hline \multirow{6}{*}{$\begin{array}{l}\text { Weight } \\
\text { (g) }\end{array}$} & F1 & $14.34 \pm 0.68^{\mathrm{b}}$ & 4.58 & $18.24 \pm 0.43^{\mathrm{cd}}$ & 1.81 & $18.06 \pm 0.30^{\mathrm{d}}$ & 2.09 \\
\hline & $\mathrm{F}_{2}$ & $13.62 \pm 0.41^{\mathrm{b}}$ & 22.28 & $49.04 \pm 1.23^{\mathrm{b}}$ & 97.74 & $15.79 \pm 0.16^{\mathrm{e}}$ & 18.92 \\
\hline & $\mathrm{BC}_{1}$ & $13.03 \pm 0.39^{b}$ & 3.19 & $13.31 \pm 0.16^{\mathrm{d}}$ & 0.49 & $25.99 \pm 0.73^{c}$ & 13.54 \\
\hline & $\mathrm{BC}_{2}$ & $15.78 \pm 1.03^{b}$ & 21.24 & $25.77 \pm 1.57^{\mathrm{c}}$ & 49.09 & $39.96 \pm 1.18^{b}$ & 13.40 \\
\hline & Mean & 16.19 & & 39.84 & & 21.11 & \\
\hline & SD & 10.59 & & 18.69 & & 10.87 & \\
\hline Seed & $\mathrm{P}_{1}$ & $379.98 \pm 17.57^{\mathrm{a}}$ & 38.86 & $285.57 \pm 17.33^{\mathrm{a}}$ & 3.40 & $160.71 \pm 16.17^{\mathrm{a}}$ & 11.69 \\
\hline Yield & $\mathrm{P}_{2}$ & $133.21 \pm 5.80^{\mathrm{bc}}$ & 33.65 & $135.60 \pm 10.28^{b}$ & 5.83 & $125.26 \pm 9.35^{\mathrm{bc}}$ & 13.50 \\
\hline \multirow{3}{*}{$\begin{array}{l}\text { per Plant } \\
(\mathrm{g})\end{array}$} & $\mathrm{F} 1$ & $110.04 \pm 3.99^{\mathrm{bc}}$ & 35.22 & $153.74 \pm 5.92^{\mathrm{b}}$ & 5.07 & $62.60 \pm 4.21^{\mathrm{d}}$ & 12.76 \\
\hline & $\mathrm{F}_{2}$ & $84.89 \pm 9.40^{\mathrm{d}}$ & 288.39 & $56.49 \pm 5.66^{\mathrm{c}}$ & 162.04 & $24.43 \pm 2.24^{\mathrm{e}}$ & 55.05 \\
\hline & $\mathrm{BC}_{1}$ & $189.57 \pm 41.57^{b}$ & 98.18 & $77.84 \pm 10.35^{\mathrm{c}}$ & 37.25 & $102.88 \pm 18.11^{\mathrm{c}}$ & 37.82 \\
\hline
\end{tabular}


QUANTITATIVE INHERITANCE OF SPIKE CHARACTERS IN CASTOR...

\begin{tabular}{|c|c|c|c|c|c|c|c|}
\hline \multirow{11}{*}{$\begin{array}{l} \\
\text { Seed Oil } \\
\text { Content } \\
(\%)\end{array}$} & $\mathrm{BC}_{2}$ & $129.20 \pm 37.90^{\mathrm{bc}}$ & 86.44 & $48.86 \pm 18.05^{\mathrm{c}}$ & 46.04 & $140.13 \pm 21.05^{\mathrm{ab}}$ & 43.30 \\
\hline & Mean & 118.22 & & 78.14 & & 57.61 & \\
\hline & SD & 138.67 & & 82.06 & & 66.63 & \\
\hline & $\mathrm{P}_{1}$ & $24.00 \pm 0.52^{\mathrm{e}}$ & 1.02 & $50.33 \pm 0.43^{\mathrm{a}}$ & 0.33 & $49.00 \pm 0.58^{\mathrm{b}}$ & 2.101 \\
\hline & $\mathrm{P}_{2}$ & $47.33 \pm 0.33^{\mathrm{a}}$ & 0.33 & $42.33 \pm 0.35^{\mathrm{c}}$ & 0.33 & $40.00 \pm 0.58^{b c}$ & 1.923 \\
\hline & $\mathrm{F} 1$ & $41.67 \pm 0.34^{\mathrm{bc}}$ & 0.33 & $48.33 \pm 0.35^{\mathrm{ab}}$ & 0.33 & $54.00 \pm 0.58^{\mathrm{a}}$ & 3.01 \\
\hline & $\mathrm{F}_{2}$ & $32.67 \pm 1.54^{\mathrm{d}}$ & 12.33 & $36.67 \pm 2.84^{\mathrm{d}}$ & 24.33 & $43.33 \pm 2.19^{c}$ & 18.33 \\
\hline & $\mathrm{BC}_{1}$ & $39.00 \pm 1.42^{\mathrm{c}}$ & 7.00 & $45.33 \pm 1.43^{\mathrm{bc}}$ & 6.33 & $46.67 \pm 1.20^{\mathrm{bc}}$ & 17.33 \\
\hline & $\mathrm{BC}_{2}$ & $44.67 \pm 2.03^{\mathrm{ab}}$ & 6.33 & $42.67 \pm 0.64^{\mathrm{c}}$ & 1.33 & $45.33 \pm 0.88^{\mathrm{bc}}$ & 15.33 \\
\hline & Mean & 38.22 & & 44.28 & & 47.39 & \\
\hline & SD & 8.28 & & 4.98 & & 3.88 & \\
\hline
\end{tabular}

The results of scaling and joint scaling tests showed inadequacy of simple additivedominance model to explain the model of gene actions for all traits evaluated in the three crosses (Table 9). At least two of the scales (A, B, C \& D) and the chi-square $\left(\mathrm{X}^{2}\right)$ for joint scaling test were significant in all the three crosses studied. The residual genetic effect $(\mathrm{m})$ was significant for all the traits studied in all the three crosses (Table 10). Significant additive effects (a) and additive $\times$ additive genes interaction (aa) were observed in the crosses for number of spikes per plant. Additive gene effects, dominance gene effects (d), additive $\times$ additive effects and dominance $\times$ dominance $(\mathrm{dd})$ effects were significant for effective length of spike in the crosses studied (Table 10). Significant dominance gene effects and significant additive $\times$ additive interaction effects were positive and significant in all the crosses for number of capsules per spike. Duplicate gene effects coupled with significant additive $\times$ dominance genes interaction (ad) were observed for weight of one hundred seeds in all the crosses (Table 10). Significant additive gene effects, dominance gene effects (d), and duplicate interactions were observed for seed yield in two out of the three crosses. Two crosses out of the three crosses recorded duplicate gene interactions for seed oil content. Only one cross recorded complementary gene interaction for the oil content (Table 10). 
BOLAJI ZULUQURINEEN SALIHU \& al.

Table 9. Scaling and Joint Scaling $(\chi 2)$ Tests for Seed Yield and Spike Characters of Three Crosses of Castor

\begin{tabular}{|c|c|c|c|c|c|c|c|c|c|}
\hline Traits & Crosses & $\mathbf{A}$ & B & $\mathbf{C}$ & D & $m$ & $a$ & $d$ & $\chi^{2}$ \\
\hline Spike per & $\mathrm{P} 1 \times \mathrm{P} 4$ & $-9.10 * * \pm 1.06$ & $1.40 \pm 1.24$ & $-11.92 * * \pm 1.95$ & $-1.83^{*} \pm 0.59$ & $5.79 * * \pm 0.19$ & $1.65 * * \pm 0.15$ & $2.04 \pm 3.37$ & $33.49 * *$ \\
\hline \multirow[t]{2}{*}{ Plant } & $\mathrm{P} 2 \times \mathrm{P} 6$ & $-2.90 * \pm 0.53$ & $-3.20 * * \pm 0.41$ & $-15.36^{* *} \pm 0.50$ & $-4.63 * * \pm 0.32$ & $3.27 * * \pm 0.07$ & $2.85^{* *} \pm 0.13$ & $10.11 * * \pm 1.90$ & $41.26^{* *}$ \\
\hline & $\mathrm{P} 5 \times \mathrm{P} 6$ & $9.70 * \pm 4.08$ & $15.90 * * \pm 0.96$ & $-0.60 \pm 0.71$ & $-12.90 * * \pm 0.65$ & $21.90 * * \pm 1.32$ & $3.60 * * \pm 0.22$ & $84.30 * * \pm 3.83$ & $83.54^{* *}$ \\
\hline \multirow{3}{*}{$\begin{array}{l}\text { Length of } \\
\text { Spike }\end{array}$} & $\mathrm{P} 1 \times \mathrm{P} 4$ & $8.50 * \pm 3.29$ & $45.40 * * \pm 4.03$ & $-5.72 \pm 5.35$ & $-29.81 * * \pm 2.57$ & $35.06 * * \pm 5.25$ & $9.65^{* *} \pm 1.08$ & $167.68 * * \pm 14.08$ & $95.61 * *$ \\
\hline & $\mathrm{P} 2 \times \mathrm{P} 6$ & $6.70 \pm 3.70$ & $8.70 \pm 4.10$ & $-46.08 * * \pm 5.96$ & $-30.74 * * \pm 3.33$ & $25.23 * * \pm 6.76$ & $8.55 * * \pm 1.18$ & $153.81 * * \pm 17.29$ & $284.77 * *$ \\
\hline & $\mathrm{P} 5 \times \mathrm{P} 6$ & $33.90 * * \pm 5.17$ & $21.80 * * \pm 2.75$ & $-45.50 * * \pm 5.66$ & $-50.60 * * \pm 2.68$ & $70.75^{* *} \pm 5.56$ & $12.95 * * \pm 1.48$ & $263.25 * * \pm 15.24$ & $139.34 * *$ \\
\hline Capsules & $\mathrm{P} 1 \times \mathrm{P} 4$ & $17.90 \pm 8.37$ & $17.90 * \pm 4.96$ & $-15.20 * \pm 6.05$ & $-25.50 * * \pm 5.32$ & $7.50 * * \pm 1.66$ & $29.49 * * \pm 14.03$ & $4.82 * * \pm 0.46$ & $123.69^{* *}$ \\
\hline \multirow[t]{2}{*}{ Per Spike } & $\mathrm{P} 2 \times \mathrm{P} 6$ & $-45.20 * \pm 12.14$ & $-62.50 * * \pm 12.93$ & $-171.64 * * \pm 19.16$ & $-31.97 * * \pm 6.90$ & $33.50 * * \pm 0.59$ & $14.85 * * \pm 2.48$ & $34.70 * * \pm 1.95$ & $280.47 * *$ \\
\hline & $\mathrm{P} 5 \times \mathrm{P} 6$ & $-66.60 * * \pm 7.20$ & $5.50 \pm 8.80$ & $-122.21 * * \pm 9.98$ & $-30.55 * * \pm 4.63$ & $110.20 * * \pm 29.85$ & $107.33 * * \pm 40.07$ & $57.73 * \pm 27.92$ & $196.15^{* *}$ \\
\hline 100 Seeds & $\mathrm{P} 1 \times \mathrm{P} 4$ & $-4.71 * \pm 1.40$ & $-41.06^{* * \pm 2.28}$ & $-48.92 * * \pm 2.44$ & $-1.57 \pm 1.38$ & $34.20 * * \pm 2.82$ & $149.42 * * \pm 5.86$ & $-35.28 * * \pm 2.87$ & $80.83^{* *}$ \\
\hline \multirow{2}{*}{ Weight } & $\mathrm{P} 2 \times \mathrm{P} 6$ & $-3.21 * * \pm 0.59$ & $-17.94 * * \pm 3.22$ & $96.85 * * \pm 5.06$ & $59.00 * * \pm 2.92$ & $-20.92 * * \pm 0.59$ & $-19.82 * * \pm 0.35$ & $-19.87 * * \pm 0.35$ & $133.14 * *$ \\
\hline & $\mathrm{P} 5 \times \mathrm{P} 6$ & $20.34 * * \pm 1.52$ & $8.53 * \pm 2.47$ & $-39.88^{* *} \pm 1.12$ & $-34.37 * * \pm 1.42$ & $-62.48 * * \pm 7.65$ & $-270.33 * * \pm 13.71$ & $150.96^{* *} \pm 8.51$ & $292.53^{* *}$ \\
\hline Seed & $\mathrm{P} 1 \times \mathrm{P} 4$ & $-110.87 * * \pm 9.69$ & $15.16 \pm 87.83$ & $-393.71 * \pm 97.45$ & $-149.00 \pm 59.31$ & $-41.40 * * \pm 8.98$ & $183.15^{* * \pm 8.44}$ & $-245.28 * * \pm 17.03$ & $736.19 * *$ \\
\hline Yield per & $\mathrm{P} 2 \times \mathrm{P} 6$ & $-283.62 * * \pm 27.64$ & $-191.61 * \pm 38.01$ & $-502.65^{* *} * 32.54$ & $-13.71 \pm 23.69$ & $123.38 * * \pm 9.25$ & $74.98 * * \pm 10.07$ & $17.72 * \pm 6.34$ & $1625.48 * *$ \\
\hline Plant & $\mathrm{P} 5 \times \mathrm{P} 6$ & $-17.54 \pm 39.88$ & $92.39 \pm 43.34$ & $-313.42 * * \pm 22.38$ & $-194.13 * * \pm 28.13$ & $353.73 \pm 349.69$ & $-477.21 * * \pm 36.37$ & $771.00 * * \pm 69.96$ & $717.18^{* *}$ \\
\hline Seed Oil & $\mathrm{P} 1 \times \mathrm{P} 4$ & $12.33 \pm 9.53$ & $0.34 \pm 2.83$ & $-23.99 * * \pm 7.25$ & $-18.33 * \pm 6.00$ & $10.00 * * \pm 1.01$ & $17.01 * * \pm 2.16$ & $36.82 * * \pm 3.12$ & $11.26^{* *}$ \\
\hline \multirow[t]{2}{*}{ Content } & $\mathrm{P} 2 \times \mathrm{P} 6$ & $-8.00 * \pm 2.52$ & $-5.32 \pm 3.37$ & $-42.64 * * \pm 4.37$ & $-14.66 * \pm 4.44$ & $11.67 \pm 9.56$ & $4.00 \pm 2.98$ & $1.50 \pm 1.21$ & $18.33^{* *}$ \\
\hline & $\mathrm{P} 5 \times \mathrm{P} 6$ & $-9.66 * \pm 3.75$ & $-9.34 \pm 4.82$ & $-29.68 * * \pm 3.24$ & $-5.34 * \pm 1.68$ & $91.99 * * \pm 5.84$ & $47.32 * * \pm 4.27$ & $8.86 * * \pm 1.56$ & $14.54 * *$ \\
\hline
\end{tabular}

* - Significant at $\mathrm{p}<0.05, * *$ - Significant at $\mathrm{p}<0.01$ 
QUANTITATIVE INHERITANCE OF SPIKE CHARACTERS IN CASTOR...

Table 10. Six Parameters-Model Estimates for Seed Yield and Spike Characters of Three Crosses of Castor

\begin{tabular}{|c|c|c|c|c|c|c|c|c|}
\hline Parameter & Crosses & $m$ & $a$ & $d$ & $a a$ & $a d$ & $d d$ & Type of Epistasis \\
\hline \multirow[t]{3}{*}{ Spike per Plant } & $\mathrm{P} 1 \times \mathrm{P} 4$ & $12.64 * * \pm 0.17$ & $-3.60 * \pm 0.49$ & $6.51 \pm 4.50$ & $3.66^{*} \pm 1.19$ & $-5.25 * * \pm 0.51$ & $4.44 \pm 2.75$ & ns \\
\hline & $\mathrm{P} 2 \times \mathrm{P} 6$ & $3.48^{* *} \pm 0.08$ & $3.30 * * \pm 0.29$ & $6.61 * * \pm 0.69$ & $9.26 * * \pm 0.66$ & $0.45 \pm 0.32$ & $-3.16 \pm 1.27$ & ns \\
\hline & $\mathrm{P} 5 \times \mathrm{P} 6$ & $6.00 * * \pm 0.11$ & $0.40 \pm 0.61$ & $24.90 * * \pm 1.33$ & $27.80 * * \pm 1.29$ & $-3.30 \pm 1.89$ & $-57.40 * * \pm 2.54$ & Duplicate \\
\hline \multirow[t]{3}{*}{ Length of Spike } & $\mathrm{P} 1 \times \mathrm{P} 4$ & $20.39^{* *} \pm 0.82$ & $-8.80 * * \pm 1.98$ & $54.17 * * \pm 5.56$ & $59.62 * * \pm 5.14$ & $-18.45^{* *} \pm 2.25$ & $-113.52 * * \pm 9.57$ & Duplicate \\
\hline & $\mathrm{P} 2 \times \mathrm{P} 6$ & $32.45^{* *} \pm 1.43$ & $7.55^{* *} \pm 5.36$ & $76.93 * * \pm 47.55$ & $61.48 * * \pm 6.39$ & $-1.00 \pm 2.76$ & $-76.88 * * \pm 11.35$ & Duplicate \\
\hline & $\mathrm{P} 5 \times \mathrm{P} 6$ & $21.65^{* * *} \pm 0.78$ & $19.00 * * \pm 2.17$ & $106.35^{* *} \pm 5.85$ & $101.20 * * \pm 5.36$ & $6.05 * \pm 2.62$ & $-156.90 * * \pm 10.36$ & Duplicate \\
\hline \multirow[t]{3}{*}{ Capsules Per Spike } & $\mathrm{P} 1 \times \mathrm{P} 4$ & $25.90^{* *} \pm 1.30$ & $33.50 * * \pm 4.64$ & $23.40 * \pm 10.83$ & $51.00 * * \pm 10.64$ & $0.01 \pm 4.67$ & $-86.80 * * \pm 19.69$ & Duplicate \\
\hline & $\mathrm{P} 2 \times \mathrm{P} 6$ & $35.11^{* *} \pm 1.73$ & $23.50 * * \pm 5.97$ & $151.09 * * \pm 16.45$ & $63.94 * * \pm 13.81$ & $8.65 \pm 6.47$ & $43.75 \pm 30.63$ & ns \\
\hline & $\mathrm{P} 5 \times \mathrm{P} 6$ & $24.04 * * \pm 0.75$ & $-1.35 \pm 4.37$ & $57.71^{* *} \pm 10.41$ & $61.11 * * \pm 9.26$ & $-36.05 * * \pm 4.79$ & $-0.02 \pm 20.15$ & ns \\
\hline \multirow[t]{3}{*}{100 Seeds Weight } & $\mathrm{P} 1 \times \mathrm{P} 4$ & $13.61^{* *} \pm 0.41$ & $-2.74 \pm 1.10$ & $-19.86^{* *} \pm 2.90$ & $3.15 \pm 2.76$ & $18.17 * * \pm 1.25$ & $42.61 * * \pm 5.05$ & Duplicate \\
\hline & $\mathrm{P} 2 \times \mathrm{P} 6$ & $49.04 * * \pm 1.23$ & $-12.46^{* *} \pm 1.57$ & $-131.18 * * \pm 5.89$ & $-118.01 * * \pm 5.85$ & $7.36 * * \pm 1.61$ & $139.15 * * \pm 8.07$ & Duplicate \\
\hline & $\mathrm{P} 5 \times \mathrm{P} 6$ & $15.79 * * \pm 0.15$ & $-13.97 * * \pm 1.39$ & $53.34 * * \pm 2.89$ & $68.75 * * \pm 2.85$ & $5.90 * * \pm 1.43$ & $-97.62 * * \pm 5.68$ & Duplicate \\
\hline \multirow[t]{3}{*}{ Seed Yield per Plant } & $\mathrm{P} 1 \times \mathrm{P} 4$ & $84.89^{* *} \pm 9.40$ & $60.37 * * \pm 6.25$ & $151.44^{* *} \pm 6.86$ & $298.00 * \pm 118.62$ & $-63.015 \pm 57.01$ & $-202.29 \pm 245.21$ & ns \\
\hline & $\mathrm{P} 2 \times \mathrm{P} 6$ & $56.49^{* *} \pm 5.66$ & $28.98^{*} \pm 10.82$ & $-29.41 * \pm 8.81$ & $27.42 \pm 47.39$ & $-46.01 \pm 23.13$ & $447.80 * * \pm 89.39$ & Duplicate \\
\hline & $\mathrm{P} 5 \times \mathrm{P} 6$ & $24.43 * * \pm 2.24$ & $-37.24 * * \pm 4.76$ & $307.88^{* *} \pm 57.18$ & $388.27 * * \pm 56.26$ & $-54.96 \pm 29.29$ & $-463.12 * * \pm 113.31$ & Duplicate \\
\hline \multirow[t]{3}{*}{ Seed Oil Content } & $\mathrm{P} 1 \times \mathrm{P} 4$ & $32.67^{* *} \pm 1.87$ & $-5.67 \pm 4.01$ & $42.67 * * \pm 3.85$ & $36.66^{* *} \pm 4.25$ & $5.99 \pm 6.02$ & $-49.33^{* *} \pm 2.15$ & Duplicate \\
\hline & $\mathrm{P} 2 \times \mathrm{P} 6$ & $36.67 * * \pm 2.22$ & $2.66 \pm 1.88$ & $31.32 * * \pm 2.01$ & $29.32 * * \pm 3.14$ & $-1.34 \pm 2.13$ & $-16.00 * * \pm 2.18$ & Duplicate \\
\hline & $\mathrm{P} 5 \times \mathrm{P} 6$ & $43.33 * * \pm 1.95$ & $1.34 \pm 0.95$ & $17.18 * * \pm 1.67$ & $10.68 * * \pm 1.19$ & $-0.16 \pm 0.92$ & $8.32 * * \pm 1.08$ & Complementary \\
\hline
\end{tabular}

Note: ns - not significant dominance effect, dominance $\times$ dominance effect or both, * Significant at $\mathrm{p}<0.05, * *-$ Significant at $\mathrm{p}<0.01$ 


\section{Discussions}

Analysis of variance (ANOVA) for the parents and their $F_{1}$ hybrids was significant in all the traits studied, indicating appropriateness of the data for genetic analysis. ANOVA for combining ability revealed significant differences of both general combining ability (GCA) and specific combining ability (SCA) for spikes/plant, effective length of spike, capsules per plant and 100-seeds weight. This is an indication of the importance of both additive and non-additive gene effects for the inheritance of the traits. RAMESH \& al. (2013) reported similar presence of both additive and non-additive gene actions in castor for all the traits studied. The high magnitude of general combining ability variances observed for spikes per plant and 100 seed weight revealed the predominance of additive gene effects for the traits and thus intra-population improvement technique is suggested for the improvement of the two traits. The result revealed non-additive gene actions (SCA) as the major contributory gene effects for the expression of effective length of spike, capsules per plant, seed yield and seed oil contents. This suggest interpopulation improvement technique for the improvement of these traits. Similar predominant variance due to SCA for the characters was reported by LAVANYA \& CHANDRAMOHAN (2003), and SOLANKI \& al. (2004) in their studies. Predominance of additive gene action in the expression of 100-seeds weight, and prevalence of non-additive gene action in the expression of seed yield, length of main spike and capsules per spike were also documented by PATHAK \& al. (1989).

From the heritability results, high to low heritability due to both broad sense and narrow sense were obtained for the traits studied among the six parents and their hybrids. High broad sense heritability and high narrow sense heritability were obtained for 100-seeds weight, and high broad sense heritability and moderate narrow sense heritability were obtained for seed oil content. This gives insight on possible genetic progress and also indicating the level of environmental influence on the traits. The high heritability indicates least influence of environment and high possible selection progress while the low heritability revealed high environmental influence. The results obtained in this study are comparable to the reports of PATEL (1991), SERVUGAPERUMAL \& al. (2000) and GOLAKIA \& al. (2007).

General combining ability effects results revealed the appropriate parent for creating specific combination desired. The present research revealed P1 as best combiners to develop castor population with high number of spikes per plant. For increase in spike length and capsules number, P2 was the best combiner for development of useful segregating population. P4 and P6 were found to be the best combiners for increase in seed weight. For seed yield per plant, P1, P4 and P5 were found to be good combiner for creating promising segregating generations. Increase in seed oil content could be achieved from good combiners such as P2 and P5. Similar reports on GCA in castor have been made by DOBARIYA \& al. (1989) and JOSHI \& al. (2001) in their work on castor. Furthermore, an efficient hybridization or breeding programme requires parents that are good combiners for a considerable number of desired characters. Therefore, it is important to assess the overall status of the parents with respect to their GCA effects over all the studied traits. The results of overall GCA status of the parents revealed that parents P1, P2 and P5 were overall good combiners. This is evident from their high overall ranking status. The implication of this result is that these parents combined their genes in a desired direction for all the studied traits, suggesting their usefulness in castor improvement programme. DOBARIYA $\&$ al. (1992) reported two out of 12 castor parents to be best general combiners for yield and yield components. GOLAKIA \& al. (2015) observed two parents to be best combiners for 
earliness to maturity and seed yield per plant. RAMESH \& al. (2013) observed one parent to be a good general combiner for most of the characters including seed yield in castor.

Based on SCA effects results, superior hybrids for different characters were identified among the crosses. The result revealed five hybrids to have significant SCA effects towards desired direction. Maximum desirable combination for spike length and capsules per spike was found in hybrid P2XP6. Desirable transgressive segregation for seed weight could be generated from P3XP5, P4XP5 and P4XP6. Desirable SCA effects for seed yield were found in eight out of the fifteen hybrids evaluated. The best combination to create population with increased seed yield was found to be P2XP6. For seed oil content, eight hybrids revealed significant desirable SCA effects with hybrids P1XP3 and P1XP5 being the best among others. All these results revealed the potentials of the hybrids for creating useful populations in later generations. Comparative findings have been documented by many researchers (NATARAJAN \& al. 1993; MEHTA, 2000; JOSHI \& al. 2001). RAMESH \& al. (2013) reported that the best performing hybrids with high mean seed yield and positive significant SCA effects for seed yield were generated from either high $\times$ average, high $\times$ high, average $\times$ average, average $\times$ high combiners for seed yield. The involvement of at least one good general combiner (as a parent) to generate good hybrid was reported by MEHTA (2000), LAVANYA \& CHANDRAMOHAN (2003), GOLAKIA \& al. (2004) and BARAD \& al. (2009). Eight hybrids namely P1XP2, P1XP3, P1XP4, P1XP5, P2XP6, P3XP5, P4XP5 and P5XP6 were good overall specific combiners for all the studied traits. These hybrids could be used for further studies and also to create promising segregating generations. Similar findings on overall specific ability have been stated by several authors [TANK \& al. 2003; SOLANKI \& al. 2004; PATEL \& CHAUHAN, 2013].

The results revealed significant heterosis over mid and better parents for most of the traits studied. Eight out of 15 hybrids evaluated showed heterosis over both mid and better parents for seed yield. For seed oil content, also eight hybrids revealed heterosis over better parents. This is an indication of presence of transgressive individuals in the hybrids evaluated. The significant heterosis over mid-parents observed in most of the crosses is a reflection involvement of non-additive gene (specific combining ability effects) for the expression of the seed yield and oil content. This is in support of the results of combining ability for the traits. The result reported here on heterosis is similar to the reports of RAMANA \& al. (2005) on three pistillate lines and nine testers of castor. Similar findings on heterosis in castor were also reported by TANK \& al. (2003), and PATEL \& PATHAK (2010).

The mean performance of the six generations, evaluated for generation mean analysis, revealed considerable inbreeding depression in all the three crosses for days to maturity, effective spike length, capsules per spike, seed yield and seed oil content. Evidence of transgressive segregation was found for spikes per plant in cross I and III, and for 100 -seeds weight in cross II. This showed the possibility of obtained better individuals for these traits in the segregating populations. Similar patterns of inbreeding depressions and transgressive segregation had been earlier reported by MANIVEL (1994) in a study in castor. Considering the $\mathrm{BC} 1$ and $\mathrm{BC} 2$ mean together, none of the backcrosses registered increased mean over their respective recurrent parents for seed yield. This is an evidence for the presence of non-additive gene effects, pointing to dominance gene action and/or epistasis interaction for the inheritance of the trait. Such complexed inheritance similar to the present findings was also reported by ANNAPPAN (1981) in a study on castor.

The results of scaling and joint scaling tests revealed that simple additive-dominance model is inadequate to elucidate the mode of gene actions for the inheritance of all the characters studied, indicating the need for analysis of digenic interaction model involving the six genetic 
parameters (m, a, d, aa, ad \& dd). Had the simple additive-dominance model with three parameters $(\mathrm{m}, \mathrm{a} \& \mathrm{~d})$ was adequate to explain the genetic system of any of the traits, none of the scales (A, B, C \& D) as well as the chi-square values would have been significant (MATHER \& JINKS, 1977). The findings reported here are related to those described by PATEL \& PATHAK (2010), and MARINKOVIC \& al. (2013). From the results, it was observed that besides the additive and dominance gene actions, epistatic interaction mechanisms have also contributed significantly to the inheritance of all the characters studied. However, the relative magnitudes of these effects varied for the characters and under different crosses evaluated. The genetic system operating for any character may be judged based on the magnitude of the six genetic parameters or judged by the magnitude and signs of dominance effect and dominance $\times$ dominance epistasis [EDWARDS \& al. 1975]. The result revealed significant effects of additive coupled with significant effects of additive $\times$ additive interaction for number of spikes per plant in two (cross I and cross II) of the three crosses. However, the effects were coupled with predominant dominance effects in all the three crosses. The simultaneous presence of significant positive additive and dominance gene actions for effective spike length suggested recurrent selection technique to be adequate in harnessing the different kinds of gene effects. For the 100seeds weight, the presence of significant additive $\times$ dominance and dominance $\times$ dominance interactions, which always reduce phenotypic dominance, would be a hindrance in simple selection technique in all the crosses. Additive gene influence was not significant for percentage seed oil content in all the crosses. The major gene effects for the trait include dominance, additive $\times$ additive and dominance $\times$ dominance gene effects. The absence of additive gene actions coupled with the presence of significant dominance $\times$ dominance effects in all the crosses would not be of advantage in simple selection procedure. However, the observed predominant dominance gene effects in the cross I and cross II presented opportunities for heterosis breeding exploitation. The significant dominance gene action, coupled with positive dominance $\times$ dominance interaction (indicating increasing dominant alleles), observed in cross III also presented similar opportunity. The results of the six parameters model reported here are similar to those reported by PATEL (1985), GONDALIYA \& al. (2001), SOLANKI \& al. (2003). PATEL \& PATHAK (2010), MARINKOVIC \& al. (2013) in their works on castor.

\section{Conclusions}

The results revealed adequate genetic variability and high potential for recombination in the parental lines evaluated. From the results, it showed that besides the additive and dominance gene actions, epistatic interaction mechanisms have also contributed significantly to the expressions of all the characters studied. High magnitude of general combining ability variance was observed for number of spikes per plant. Non-additive gene actions were found to be the major contributory gene effects for the expression of effective length of spike, seed yield and seed oil contents. However, varietal differential was observed on relative magnitudes of the gene effects for the characters under study.

The present research findings suggest that improvement of the traits evaluated may not be easily achievable by adopting simple selection technique, rather heterosis breeding and population improvement involving inter mating among promising divergent genotypes may be more appropriate. 


\section{Notes on contributors}

Bolaji Zuluqurineen SALIHU (PhD) is a research scientist and a geneticist with a special interest in quantitative genetics. His work majorly focuses on castor population improvement.

Isiaka Amuda YUSUF (PhD) and Muhammad Nur ISHAQ are agronomists who has interest in agronomy of castor.

Benson Obukohwo APUYOR and Abolore Adijat AJADI are research scientists with research interest in castor processing and utilization. Their work including screening of castor germplasm for high seed oil content and good physicochemical properties

Maryam Alfa KABARAINI is a researcher who has research interest in castor disease management.

\section{References}

ANNAPPAN R. S. 1981. Genetic analysis in castor (Ricinus communis L.). PhD thesis, Tamil Nadu Agricultural University, Coimbatore, India.

BARAD Y. M., PATHAK A. R. \& PATEL B. N. 2009. Heterosis for yield and yield attributing traits in castor, Ricinus communis L. Journal of Oilseeds Resources. 26(2): 102-104.

DAVID S. M. 2006. Genetic studies on seed coat texture and cooking time in some varieties of cowpea (Vigna unguiculata (L.) Walp.). PhD thesis, University of Jos, Jos, Nigeria.

DOBARIYA K. L., PATEL I. D., PATEL P. S. \& PATEL V. J. 1989. Combining ability and genetic architecture of oil content in castor (Ricinus communis L.). Journal of Oilseeds Research. 6(1): 92-96.

DOBARIYA K. L., DANGARIA C. J. \& PATEL V. J. 1992. Combining ability analysis in castor. Journal of Maha. Agricultural Universal. 17(1): 235-238.

EDWARDS L. H., KETATA H. \& SMITH E. L. 1975. Gene Action of Heading Date, Plant Height, and Other Characters in Two Winter Wheat Crosses. Crop Science. 16(1): 275-277.

GANA A. K., YUSUF A. F. \& APUYOR B. 2013. Castor oil plant and its potential in transformation and industrialization of under developing nations in the world. Advanced Journal of Agricultural Research. 1(5): $72-79$.

GOLAKIA P. R., MADARIA R. B., KAVANI R. H. \& MEHTA D. R. 2004. Gene effects, heterosis and inbreeding depression in castor. Journal of Oilseeds Research. 21(2): 270-273.

GOLAKIA P. R., KAVANI R. H. \& MONPARA B. A. 2007. Genetic variation and trait relationship in castor. National Journal of Plant Improvement. 9(1): 60-62.

GOLAKIA P. R., POSHIYA V. K. \& MONPARA B. A. 2015. Identification of superior donor parents for earliness through combining ability in castor. International Journal of Research in Plant Science. 5(3): 26-31.

GONDALIYA A. B., DANGARIA C. J., KAVANI R. H. \& GOLAKIA P. R. 2001. Genetic architecture for yield and its components in castor. Journal of Oilseeds Research. 18(2): 150-153.

GRIFFING B. 1956a. A generalized treatment of diallel crosses in quantitative inheritance. Heredity. 10: 31-50.

JOSHI H. R., MEHTA D. R. \& JADON B. S. 2001. Heterosis of yield and yield components in castor hybrids. Journal of Oilseeds Research. 18(2): 164-169.

LAVANYA C. \& CHANDRAMOHAN Y. 2003. Combining ability and heterosis for seed yield and yield components in castor. Journal of Oilseeds Research. 20(2): 220-224.

MANIVEL P. 1994. Heterosis, combining ability, generation mean and stability analysis in castor (Ricinus communis L.). PhD thesis, Tamil Nadu Agricultural University, Coimbatore, India.

MARINKOVIC R., SKLENAR P., MARJANOVIC-JEROMELA A., MILADINOVIC D., JANKULOVSKA M., DUSANIC \& MIKIC N. A. 2013. Components of genetic variance for some quantitative characteristics in castor bean. Bulgarian Journal of Agricultural Science. 19(1): 1225-1230.

MATZINGER D. F. \& KEMPTHORNE O. 1956. The modified diallel table with partial inbreeding and interactions with environment. Genetics. 41: 822-833.

MATHER K. \& JINKS J. L. 1977. Introduction to Biometrical Genetics. London, UK: Chapman and Hall Ltd. 41 pp.

MEHTA D. R. 2000. Combining ability analysis for yield and its component characters in castor (Ricinus communis L.). Indian Journal of Agricultural Research. 34(1): 200-212.

OGUNNIYI D. S. 2006. Castor Oil: A vital industrial raw material. Bioresource Technology. 97(9): 1086-1091.

PATEL K. M. 1985. Heterosis, combining ability and gene action in castor (Ricinus communis L.). MSc thesis, Gujarat Agricultural University, Nagar, India.

PATEL N. H. 1991. Genetic analysis of yield and its components in castor (Ricinus communis L.). PhD thesis, Rajasthan Agricultural University, Bikaner Campus, Udaipur, India. 
PATEL P. B. \& PATHAK H. C. 2010. Genetic architecture for yield and its composition in castor (Ricinus communis L.). International Journal of Agricultural Sciences. 6(2): 418-421.

PATEL M. S. \& CHAUHAN R. M. 2013. Study on gene action, heterosis and inbreeding depression for yield and quality traits in castor (Ricinus communis L.). Journal of Oilseeds Research. 30(1): 138-143.

PATHAK H. C., DIXIT S. K. \& PATEL P. G. 1989. Line x tester analysis for seed yield and its components in castor (Ricinus communis L.). Indian Journal of Genetics. 49(1):125-129.

PBTOOLS-1.3. 2014. Biometrics and Breeding Informatics, PBGB Division, International Rice Research Institute, Los Baños, USA, Laguna.

RAMANA P. V., LAVANYA C. \& RATNASREE P. 2005. Combining ability and heterosis studies under rainfed conditions in castor (Ricinus communis L.). Indian Journal of Genetics and Plant Breeding. 65(1): 325-326.

RAMESH T. \& VENKATE S. 2001. Path coefficient analysis in castor. Agricultural Science Digest. 21(1): 59-60.

RAMESH M., LAVANYA C., SUJATHA M., SIVASANKAR A., ARUNA-KUMARI J. \& MEENA H. P. (2013). Heterosis and combining ability for yield and yield component characters of newly developed castor (Ricinus communis 1.) hybrids. Journal of Life Sciences. 8(4): 1421-1424.

RAO P. V. R., GIRI V. G. \& PACHAURI D. K. 2006. Evaluation of castor (Ricinus communis L.). Research on Crops. 1(1): 699-708.

SAKHARE S. B., PRIYA P., UDASI R. N. \& NAGDEVE M. B. 2017. Genetic analysis of yield and its biometric traits in castor (Ricinus communis L.). Electronic Journal of Plant Breeding. 8(1): 78-83.

SERVUGAPERUMAL S., RANGASWAMY P. \& MUPPIDATHI N. 2000. Genetic variability, correlation and path coefficient analysis in castor. Madras Agricultural Journal. 86(1): 456-459.

SHIVANNA S. 2008. Genetic diversity, combining ability and stability analysis of selected castor lines. PhD Thesis, University of Agricultural Sciences, Bangalore, India.

SOLANKI S. S., JOSHI P., GUPTA D. \& DEORA V. S. 2003. Gene effects for seed yield contributing characters in castor (Ricinus communis L.) by generation mean analysis. Journal of Oilseeds Research. 20(1): 217-219.

SOLANKI S. S., DEORA V. S. \& SINGH D. P. 2004. Combining ability of new castor (Ricinus communis L.) pistillate lines: MCP-1-1, Journal of Oilseeds Research. 21(1): 274-276.

STAR-2.0.1. 2014. Biometrics and Breeding Informatics, PBGB Division, International Rice Research Institute, Los Baños, USA, Laguna.

TANK C. J., JAIMINI S. N. \& RAVINDRABABU Y. 2003. Combining ability analysis over environments in castor (Ricinus communis L.). Crop Research. 26(1): 119-125.

WEISS E. A. 2000. Oilseed crops. Oxford, England: Blackwell Science, 39 pp.

How to cite this article:

SALIHU B. Z., YUSUF I. A., APUYOR B. O., AJADI A. A., KABARAINI M. A. \& ISHAQ M. N. 2018. Quantitative inheritance of spike characters in castor (Ricinus communis L.). J. Plant Develop. 25: 59-75. https://doi.org/10.33628/jpd.2018.25.1.59 